\title{
Consumo, hábitos alimentarios y habilidades culinarias en alumnos de tercero a quinto año básico y sus padres
}

\author{
Food consumption, dietary behaviour \\ and cooking skills in third to fifth grade school-age \\ children and parents
}

\begin{abstract}
The objective of this study was to describe food consumption, dietary behaviour and culinary habits in third to fifth grade students and their families through surveys and focus groups to implement nutrition education interventions in healthy eating habits at schools. Surveys in parents and children demonstrated that healthy food consumption was under the Chilean Dietary Guidelines recommendatiolns. Consumption of fruits, vegetables and dairy food in parents was less than in children, but nonhealthy food consumption was also less in parents. In dietary behavior $22 \%$ of children consumed breakfast at home and also at school, and 33\% consumed double lunch. Snack was carried from home in $60 \%$ of children, but $72 \%$ carried money to buy non-healthy snacks at school. Parents said that they had norms related to dietary behavior at home, but $60 \%$ watched TV during dinner and $96 \%$ had afternoon tea and just $42.2 \%$ had dinner. Regarding culinary habits, students had the skills to prepare simple food but did not have the skills to prepare a dish. The mothers said they had the culinary skills but they cook simple food like pasta, rice and potatoes. Focus groups confirmed survey findings, identifying three profiles in mothers and separating eating behaviour during the weekdays and weekend. Its notorious that mothers consuming a healthy diet were those who had more health problems. Barriers to healthy eating were lack of time and money. Surveys and focus groups showed a good access by parents and children to Information and Communication Technologies. In conclusion, it is urgent to design nutrition education programs to create healthy eating habits considering the current situation in food consumption, dietary behaviour and culinary habits in students and their parents.

Key words: food consumption, dietary behaviour, culinary habits, nutrition education, focus grups, third to fifth grade students, parents.
\end{abstract}

\section{INTRODUCCIÓN}

Existe consenso entre investigadores, educadores, personal de salud y autoridades políticas de la necesidad urgente de introducir los temas de alimentación y nutrición en las escuelas básicas del país para educar a los alumnos y sus familias en conocimientos, hábitos alimentarios y prácticas de alimentación saludable para prevenir la obesidad, contribuyendo a mejorar su salud y calidad de vida en la edad adulta (1). Para ello se considera a la escuela, y en ella a los docentes, como agentes
Fernando Vio del R(1) Lydia Lera M. (1)

Carmen Gloria González G. (1)

Marisol Yáñez G.(2)

Gabriela Fretes C. (3)

Edith Montenegro V. (4)

Judith Salinas C (1)

(1) Instituto de Nutrición y Tecnología de los Alimentos Dr. Fernando M onckeberg Barros, (INTA), Universidad de Chile, Santiago, Chile.

(2) Programa Vida Sana, Corporación de Desarrollo Social de Providencia, Santiago. (3) Unidad de Prevención de la O besidad, Dirección de Vigilancia de Enfermedades No Transmisibles,

Ministerio de Salud Pública y Bienestar Social, Paraguay. (4) CESFAM Centenario, Servicio de Salud Aconcagua, Chile.

Dirigir la correspondencia a: Dr. Fernando Vio del Rio Instituto de Nutrición y Tecnología de los Alimentos Dr. Fernando M onckeberg Barros, (INTA) Universidad de Chile, Santiago, Chile. Av. El Líbano 5524 - Macul Fono: 229781576

E-mail: fvio@inta.uchile.cl

Este trabajo fue recibido el 10 de Julio de 2015 y aceptado para ser publicado el 5 de Octubre de 2015 .

fundamentales en la promoción y fomento de la salud de la comunidad educativa, así como la importancia que tiene la cotidianidad de la escuela en el desarrollo de estilos de vida saludables. Esto no incluye sólo a los niños y maestros, sino también a sus familias y comunidades de las que forman parte (2). En las instituciones educacionales existe la necesidad de contar con materiales educativos adecuados y metodologías docentes sencillas para abordar esta temática, lo cual no está presente en las políticas actuales del M inisterio de Educación 
ni en sus normas y regulaciones (3), a pesar de múltiples experiencias realizadas en el país para enfrentar este tema, dada la alta y creciente prevalencia de la obesidad infantil (4). Para enfrentar lo anterior, existe una línea de investigación de educación en alimentación y nutrición desde el Proyecto Fondecyt №1110044, “Elaboración, aplicación y evaluación de un programa de intervención con materiales educativos en alimentación saludable para profesores, alumnos de prebásica, básica y sus familias", realizado entre los años 2011 y 2013, que investigó el tema en niños de prekinder, kínder, primero y segundo año básico con metodologías participativas y uso de las Tecnologías de la Información y Comunicación (TIC). Esta línea de investigación se continuó con un nuevo proyecto (2014-1016) en niños de tercero a quinto básico (5). En el primer año de este proyecto, se realizó un estudio descriptivo sobre consumo, hábitos alimentarios y habilidades culinarias en los alumnos de tercero a quinto básico y sus padres, con encuestas y grupos focales, para posteriormente elaborar un modelo educativo integral en alimentación saludable.

El objetivo de este estudio fue describir el consumo de alimentos, los hábitos alimentarios y las habilidades culinarias en alumnos de tercero a quinto año básico y de sus familias por medio de encuestas y grupos focales para realizar intervenciones educativas en alimentación saludable en el ámbito escolar.

\section{SUJETOS Y M ÉTODOS}

Diseño del estudio

Estudio descriptivo cuali-cuantitativo, de corte transversal acerca de consumo, hábitos alimentarios y habilidades culinarias en alumnos y padres de tercero a quinto año básico de nivel socioeconómico medio bajo, asistentes a escuelas particulares subvencionadas, con un Índice de Vulnerabilidad Escolar (IVE) del M inisterio de Educación superior a 70, Io cual significaba que más del $70 \%$ de los niños eran pobres y requerían de asistencia alimentaria.

\section{Sujetos}

M uestra de niños y padres para encuestas

El cálculo de la muestra se realizó en base a la estimación de la media del zIMC de 1,0, con una desviación estándar de 1,12 , que se obtuvo del proyecto "Prevención y control de la obesidad infantil de M acul para escolares de 1ํa 4으 básico" (6), con un nivel de significación del 5\% y un nivel de confianza del $90 \%$, dando como resultado una muestra de 90 niños (30 alumnos por nivel, 43 niños y 47 niñas), con sus respectivos padres (24 hombres y 66 mujeres). Este cálculo se hizo en forma similar a lo realizado para calcular la muestra del proyecto Fondecyt № 1110044 para hacer el diagnóstico del conocimiento y consumo alimentario de escolares de prekinder a segundo año básico (7). Para el cálculo se utilizó el software STATA 14.0 para W indows (8). Las encuestas se realizaron en una escuela particular subvencionada de San Felipe.

\section{M uestra de grupos focales a padres}

La técnica escogida para la realización de esta investigación fue el muestreo intencionado (9). El criterio de inclusión para la selección de la muestra consistió en seleccionar padres de tercero a quinto año básico en colegios particulares subvencionados de M elipilla y San Felipe. La muestra quedó constituida por 26 madres que estuvieron dispuestas e interesadas en participar en una investigación. La muestra de los cuatro grupos focales, dos en M elipilla y dos en San Felipe, tuvo un número mínimo de 5 y máximo de 8 participantes de los tres cursos mencionados, lo cual está de acuerdo con el número de participantes requerido por Aschidamini\& Saupe (10).

\section{Encuestas a niños y padres}

Para realizar la encuesta no se contaba con un instrumento para investigar conocimientos, consumo, hábitos alimentarios y habilidades culinarias en niños de 8 a 11 años. Para ello, fue necesario diseñar y validar una encuesta siguiendo la metodología de evaluación de indicadores aplicada en el proyecto anterior para familias de escolares de 4 a 7 años (11). Esta validación demostró que en niños de 8 a 11 años no se justificaba hacer preguntas de conocimiento, ya que prácticamente todos conocían las Guías Alimentarias Chilenas (12) y respondieron adecuadamente, sin discriminar, las 12 preguntas de conocimiento. Por esta razón, estas preguntas se eliminaron de la encuesta original quedando la encuesta definitiva con 28 preguntas: 12 de frecuencia de consumo, 10 sobre hábitos alimentarios y 6 de habilidades culinarias y gastos en la escuela (13). Para los padres se aplicó una encuesta validada en un proyecto anterior (14).

\section{Análisis estadístico encuestas niños y padres}

Se realizó un análisis descriptivo de variables hábitos de alimentación y consumo de alimentos según género, para los niños y padres, por medio de porcentajes y por una distribución percentilar. Se utilizó el test de Mann-Whitney para la comparación de las variables de consumo por sexo. El nivel de significación estadística para esta investigación se estableció como 0,05. Para el análisis se utilizó el software estadístico STATA 14.0 para W indows (8).

\section{Grupos focales a padres}

La técnica de grupos focales tiene por finalidad la producción controlada de un discurso por parte de un grupo de sujetos que se reúnen durante un espacio de tiempo limitado, con el fin de debatir sobre un determinado tópico propuesto por el investigador (15). La información oral fue transcrita a textos y posteriormente se trató mediante la técnica de "Análisis de Contenido", que permite leer e interpretar documentos escritos utilizando un procedimiento analítico orientado a dar sentido a lo estudiado. Se trata de una interpretación donde el dato puede poseer un sentido simbólico manifiesto o latente, es decir, no posee un significado único. Asimismo, puede ser emitido de forma consciente/inconsciente por parte del entrevistado. El esquema de análisis de contenido que se siguió para procesar los datos fue el propuesto por Navarro y Díaz (16).

\section{Análisis de la información grupos focales}

La parte final del procedimiento de análisis de los datos fue la interpretación de las categorías obtenidas, la cual se desarrolló siguiendo los criterios fijados según el nivel de profundidad, a través de la agrupación de respuestas y establecimiento de discursos por segmento. Esta metodología busca significados que se reagrupan en categorías, lo que refleja las ideas fuerza más relevantes e importantes. Los resultados se valoraron según las opiniones e ideas fuerza, definiendo tres perfiles: el Perfil 1 es el grupo mayoritario; el Perfil 2 es el grupo intermedio y el Perfil 3 es el grupo minoritario. Esto tiene directa relación con la cantidad de opiniones expresadas en los grupos focales, que se describen a continuación:

- Perfil 1: comprende el grupo predominante, en donde las categorías aparecen claramente dentro de las experiencias individuales y se pueden aunar en un discurso claro y 
certero. Está integrado por "madres que son dueñas de casa".

- Perfil 2: representa el grupo intermedio, cuyas ideas representan una realidad que irrumpe con fuerza frente al 1 y 3. Está integrado por "madres que trabajan fuera de casa".

- Perfil 3: se entiende por las ideas que aparecen con menos fuerza, pero representan realidades importantes. Desde la perspectiva sociológica, este grupo rompe el consenso social que se desprende de los otros perfiles, con propuestas contrarias a la norma mayoritaria. Las ideas de este grupo no siempre influyen, pero generan nuevos planteamientos. Este grupo es heterogéneo, integrado por madres dueñas de casa y trabajadoras fuera de casa.

El estudio fue aprobado por el Comité de Etica del INTA de la Universidad de Chile. Cada padre o madre firmó un consentimiento informado, donde se describían los objetivos y características del estudio para él y para su hijo. Además, cada niño firmó un asentimiento.

\section{RESULTADOS}

Encuestas a niños

En la tabla 1 se observa que el consumo de alimentos saludables está por debajo de lo recomendado en las Guías Alimentarias (12). La mediana de vasos de agua diarios fue
3 , siendo la recomendación de 6 a 8 vasos al día; la mediana de frutas 2,5; la de productos lácteos 2 y la de pescado 1, por debajo de lo recomendado, que se cumplía sólo para verduras y legumbres. El resto de los alimentos, considerados como no saludables, presentan un bajo consumo. Los alumnos hombres comían significativamente más pan, bocadillos salados y dulces, jugos y bebidas gaseosas que las mujeres.

En hábitos alimentarios Ilamó la atención que 22\% tomaba doble desayuno y 33,3\% doble almuerzo, es decir, en la casa y en el colegio. El 72,2\% de los niños llevaba dinero a la escuela para comprar alimentos, que dicen ser fruta $(66,3 \%)$, galletas $(61,3 \%)$, bebidas y jugos azucarados $(56,3 \%)$, leche 0 yogur $(52,5 \%)$, dulces, chocolates. Un $60 \%$ Ilevaba colación de la casa y además dinero.

En habilidades culinarias, $70 \%$ dijo preparar ensaladas 1 a 4 días por semana, 66,6\% hacer un sándwich y $46,7 \%$ pelar y picar fruta. Sólo un tercio de los niños dijo ser capaz de preparar un plato de comida sin ayuda, siendo la mitad de las preparaciones arroz y la otra mitad fideos y huevos.

\section{Encuestas a padres}

En la tabla 2 se describe el consumo de alimentos en los padres, en donde a diferencia de los hijos, consumen sólo 1 porción de frutas, verduras y lácteos, manteniendo las 2 porciones de legumbres y 1 de pescado de sus hijos. Los

TABLA 1.

Consumo de alimentos en niños de 8 a 11 años por sexo

\begin{tabular}{|c|c|c|c|}
\hline Consumo P50 (P25-P75) & $\begin{array}{l}\text { Hombres } \\
n=43\end{array}$ & $\begin{array}{c}\text { M ujeres } \\
n=47\end{array}$ & $\begin{array}{l}\text { Total } \\
\mathrm{n}=90\end{array}$ \\
\hline Verduras/Ensaladas (porciones al día)* & $2(1-2)$ & $2(1-2)$ & $2(1-2)$ \\
\hline Frutas (porciones al día) & $3(2-3)$ & $2(2-3)$ & $2.5(2-3)$ \\
\hline $\begin{array}{l}\text { Lácteos (leche descremada, quesillo, yogurt) } \\
\text { (porciones al día) }\end{array}$ & $2(1-3)$ & $2(2-3)$ & $2(2-3)$ \\
\hline Pescado (veces por semana) & $1(1-1)$ & $1(1-1)$ & $1(1-1)$ \\
\hline $\begin{array}{l}\text { Legumbres como lentejas, arvejas, porotos o garbanzos? } \\
\text { (veces por semana) }\end{array}$ & $2(1-3)$ & $2(1-2)$ & $2(1-2)$ \\
\hline Pan (unidad) *** & $2(1-2)$ & $1(1-1)$ & $1(1-2)$ \\
\hline $\begin{array}{c}\text { Comidas rápidas preparadas } \\
\text { (pizza, hamburguesas, completos, empanadas, sopaipillas) } \\
\text { (veces por semana) }\end{array}$ & $1(1-1)$ & $1(1-1)$ & $1(1-1)$ \\
\hline $\begin{array}{l}\text { Bocadillos dulces (helados, galletas, golosinas) } \\
\text { (veces a la semana)** }\end{array}$ & $1(1-2)$ & $1(1-1)$ & $1(1-2)$ \\
\hline $\begin{array}{l}\text { Bocadillos salados (ramitas, papas fritas, nachos) } \\
\text { (veces a la semana)** }\end{array}$ & $2(1-2)$ & $1(1-2)$ & $1(1-2)$ \\
\hline $\begin{array}{l}\text { Pasteles y masas dulces como tortas, queque, Berlín } \\
\text { (veces a la semana) }\end{array}$ & $1(1-1)$ & $1(1-2)$ & $1(1-2)$ \\
\hline Jugos y/o bebidas gaseosas (vasos al día) ** & $2(1-3)$ & $1(1-2)$ & $2(1-2)$ \\
\hline Agua (vasos al día) & $3(2-3)$ & $2(2-3)$ & $3(2-3)$ \\
\hline
\end{tabular}

Two-sample Wilcoxon rank-sum (M ann-W hitney) test: ${ }^{*} p<0.05 ;{ }^{* * p}<0.01 ; * * * p<0.001$ 
hombres comen más legumbres y menos bocadillos dulces que las mujeres (diferencia no significativa). En comida rápida, las mujeres tienen una tendencia a comer más: 75\% dice consumir a lo menos una comida rápida en la semana; en cambio, $75 \%$ de los hombres reportaron no consumir. Llama la atención el bajo consumo de comidas no saludables como comidas rápidas, bocadillos dulces y salados, jugos y bebidas azucaradas, lo cual es mucho menor que en los niños, como se aprecia al compararlos en la figura 1.

En hábitos, los padres señalaron que 78,9\% comen jun-

TABLA 2.

Consumo de alimentos en padres de niños de 8 a 11 años por sexo

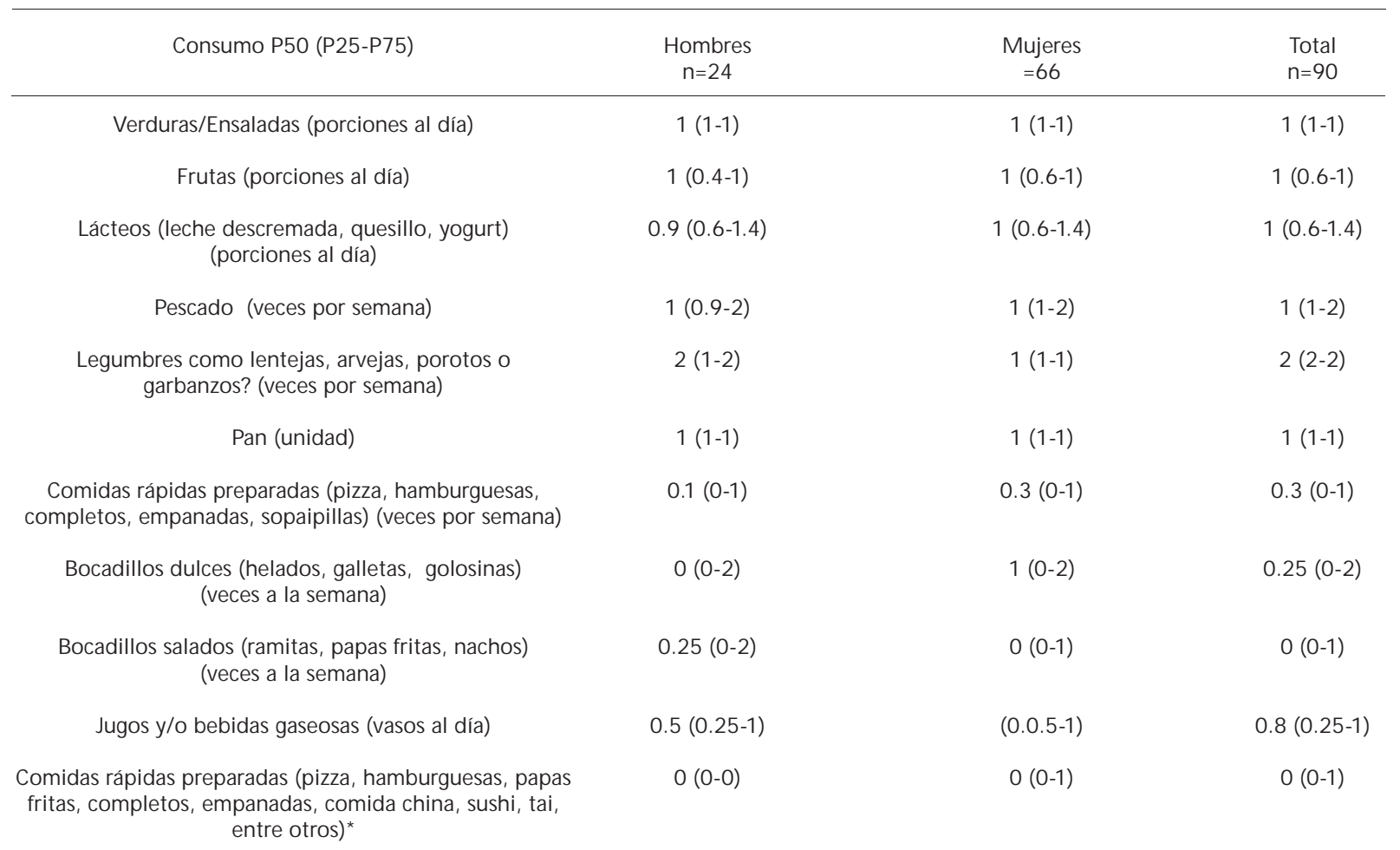

Two-sample Wilcoxon rank-sum ( $M$ ann-W hitney) test: $* \mathrm{p}=0.07$

Comparación del consumo alimentario de padres y niños.

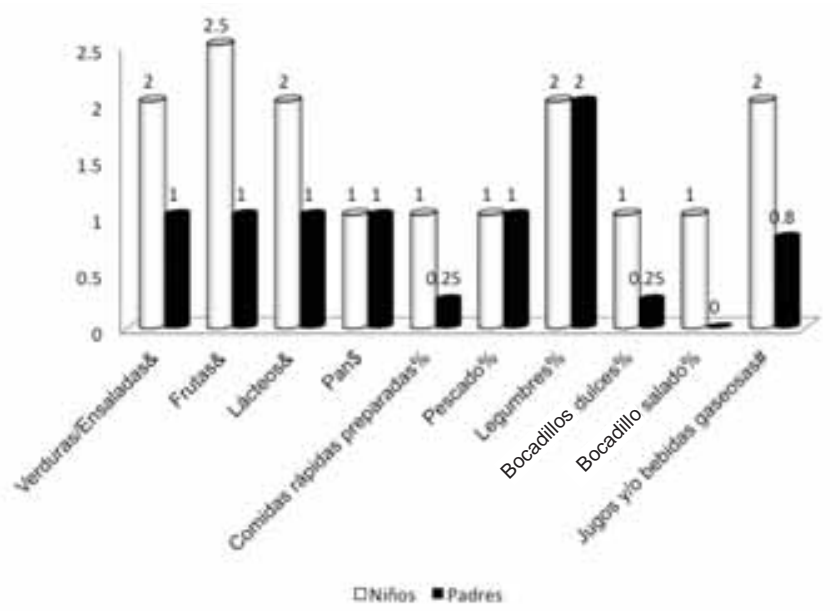

Leyenda: \&Porciones al día, \$unidad, \%veces por semana, \#vasos al día 
tos, $60 \%$ ve TV cuando comen y $81,1 \%$ dicen tener normas de alimentación en la casa. Un $95,6 \%$ toma once, contra un $76 \%$ que dicen desayunar y almorzar, por lo cual la once pasa a ser la principal comida del día, ya que sólo 42,2\% dice comer en la noche.

De los que comían fuera del hogar (60\%), más de la mitad lo hace en restaurantes (68\%), prefiriendo comida casera, y sólo $11 \%$ lo hace en locales de comida rápida.

En habilidades culinarias, la comida en casa la prepara la mamá $(93,3 \%)$ y $82,2 \%$ dice que le gusta cocinar. En cuanto a preparación de alimentos, 79\% dice preparar ensaladas verdes diariamente, pero sólo 14,4\% prepara cena con pollo, pescado, vegetales o una cena completa para dos personas todos los días (18,9\%).

Además, a los padres se preguntó el uso de TIC, encontrándose que $100 \%$ cuenta con TV y celular y $83,3 \%$ tiene computador en el hogar, con acceso a internet $(65,6 \%)$ y videojuegos $(43,3 \%)$.

Lo que más utilizan los padres son las redes sociales $(62,7 \%)$, seguido de mensajes de texto $(40 \%)$ y correo electrónico (32\%). En la escuela, $92 \%$ de los niños tiene acceso al computador. Para estudiar, los niños utilizan en primer lugar la información verbal de los padres (93,3\%), seguido por libros revistas o enciclopedias $(83,3 \%)$ y portales de internet $(80 \%)$.

\section{Grupos focales madres}

Las preguntas que guiaron la conversación en los grupos focales se presentan en la tabla 3. Los hábitos de alimentación al desayuno almuerzo y comida están descritos en la tabla 4 y lo que sucede los fines de semana en tabla 5. El grupo mayoritario (perfil 1) está constituido por madres dueñas de casa con poca información de cómo alimentarse en forma adecuada, sin un orden en las comidas, ya que se dejan llevar por la rutina de sus hijos los días de semana. Aun cuando almuerzan comida casera, comen casi siempre lo mismo, con muy poca innovación. El grupo intermedio (perfil 2) son madres que trabajan, tienen poco tiempo para cocinar y por lo tanto utilizan comida fácil de preparar, como arroz, fideos y puré durante la semana. El grupo minoritario (perfil 3) se alimenta bien y se preocupa de la comida de los hijos. En general, corresponde a madres que han tenido algún problema de salud y contacto con nutricionista, razón por la cual cambiaron su dieta poco saludable por una saludable.

Como se ha visto en otros estudios de hábitos en la población chilena (17-20), la mayor parte de las familias toman té $u$ once y no cenan en la noche.

Los fines de semana cambian los hábitos y familias que tienen malos hábitos durante la semana hacen un esfuerzo por mejorar los fines de semana, al tener más tiempo para cocinar. En cambio, otros se relajan y tienden a comer alimentos poco saludables el fin de semana.

En cuanto al almuerzo fuera del hogar (tabla 6), los adultos llevan comida desde la casa y los hijos comen en el colegio y vuelven a comer en su casa cuando regresan en la tarde, lo cual

TABLA 3.

Temas y preguntas grupos focales apoderados

Tema

Hábitos de alimentación personal

Actitudes y conductas

Habilidades culinarias y autoeficacia

TICS Tecnologías de Información y Comunicación

Fortalezas y necesidades
Preguntas

Comente en general acerca de sus hábitos de alimentación personal

Si usted pudiera evaluar sus hábitos de alimentación personal, con qué nota se calificaría del 1 al 7 Acerca de su alimentación los días de trabajo, comente acerca de qué comidas tienen en el colegio (desayuno, almuerzo, bocadillo) y comente acerca del tiempo y lugar en donde las realizan Podrían contarnos brevemente cómo es su alimentación los días de fin de semana

Comente esta frase:

"Alimentarse de forma saludable es muy importante, pero en la práctica es muy difícil". "Alimentarse de manera saludable es muy caro"

Qué opinan de las etiquetas nutricionales que aparecen en los diversos alimentos, cuéntennos si las leen, en qué se fijan y a su vez si las comprenden

¿Cómo definirían sus habilidades para realizar un almuerzo o cena saludable? En el caso de responder "No", diga cuáles son sus mayores barreras

Han encontrado alguna dificultad a la hora de pensar ¿qué cocino hoy?... ¿Alguno/a de ustedes tiene menú semanal en casa?

Sin importar si es la persona que cocina en casa, que nota se pondría como cocinero, del 1 al 7 ¿Cuáles son las principales fortalezas y dificultades para ser un buen cocinero?

¿Qué opinan de que los niños sean más activos en la cocina? ¿Qué les parece la idea de incluir a los niños en la cocina y enseñarles diversas herramientas en el aula? ¿Cómo resultaría? ¿Qué les parecen los talleres de cocina? Comente

¿Qué opinan acerca de la tecnología y los niños? En especial queremos saber tanto lo positivo como lo negativo

¿Cuál es el medio tecnológico que más utilizan ustedes, ej. celulares, tablet, computadores? ¿Con qué frecuencia utilizan internet?

¿Alguna vez han utilizado la tecnología en la cocina? y ¿cómo les ha resultado esa experiencia?

¿Cuáles creen que son sus mayores fortalezas para implementar un programa de alimentación saludable?

Diga en qué temas específicos les gustaría ser capacitados acerca de alimentación saludable, y bajo qué modalidad 
se vio en las encuestas en un tercio de los casos. Después los niños toman once y no cenan en la noche. Un grupo menor de padres va a almorzar a su casa y otro compra la comida en el trabajo, porque no se la dan ni llevan de su casa.

Al ponerse nota sobre sus hábitos alimentarios, el promedio es 4,4 (rango de 1 a 7), lo que refleja una percepción de que se alimentan mal porque tienen poco tiempo para cocinar y alimentarse adecuadamente.

Los padres relatan que para ellos es muy difícil controlar la alimentación de sus hijos que están en la pre adolescencia, ya que comen en el colegio y además les dan dinero (\$300 a $\$ 500$ diarios).

Según los padres, existen dos barreras para alimentarse en forma saludable: la primera es que los productos saludables son más caros, en especial verduras, frutas, pescados y lácteos sin azúcar ni grasas y por lo tanto prefieren arroz o fideos antes que comprar frutas o verduras. La segunda es porque se necesita mucho tiempo para hacer una preparación saludable, porque son platos más elaborados y complejos.

En general, no saben leer las etiquetas nutricionales para escoger alimentos más saludables y compran según el precio o las preferencias de la familia, especialmente de los niños, muchas veces influidos por la publicidad. Solamente personas que han tenido algún problema de salud y contacto con nutricionistas, saben leer etiquetas, pero muchas veces reconocen seguir comprando alimentos poco saludables.

En habilidades culinarias, la mayoría se siente capaz de realizar comidas saludables, pero no lo hacen por las barreras de precio y tiempo ya descritas, a las que se agregan falta de creatividad y dificultad para darle en el gusto a cada uno de los integrantes de la familia. Las madres que trabajan cocinan en la noche o para varios días. Las dueñas de casa lo hacen todos los días porque tienen tiempo, pero no tienen la suficiente información para diversificarse e innovar, ni para organizar el tiempo y dinero para las compras. Existe un grupo más pequeño al que no le interesa cocinar. En general, se califican muy bien en sus habilidades para cocinar, con nota 6 en la escala 1 a 7.

Los padres coinciden en que a niños y niñas les gusta cocinar, pero sienten temores frente a ello, por lo cual los evitan en la cocina y prefieren que sólo realicen preparaciones frías. Sin embargo, valoran estar con los niños en la cocina porque se produce una interacción positiva.

Respecto a las TIC en sus hijos, encuentran positivo que se manejan muy bien con ellas y que son muy útiles para obtener información en las tareas escolares, pero ven como negativo que siempre jueguen con ellas, sin respetar los límites que tratan de poner los padres.

En cuanto a los medios tecnológicos más utilizados por los padres, predomina el celular con o sin internet, diferenciándose en su uso las madres menores de 40 años de las mayores, ya que las jóvenes las usan con mayor frecuencia para distracción, trabajo, redes sociales y también para ver recetas de cocina. Al consultársele sobre los programas de alimentación para sus hijos a través de las TIC, la mayoría está de acuerdo porque a los niños les gusta la tecnología para "aprender jugando", por

TABLA 4.

Hábitos de alimentación de los apoderados en días de semana según perfiles.

\begin{tabular}{lll}
\hline Perfil 1 & Perfil 2 \\
\hline Desayuno & Desayunan con poco tiempo & $\begin{array}{l}\text { No toman desayuno en su casa, por } \\
\text { falta de tiempo, trabajo, estudio } \\
\text { o dieta }\end{array}$ \\
$\begin{array}{l}\text { Generalmente toman té o leche y } \\
\text { comen pan con acompañamiento sin } \\
\text { considerar otros alimentos }\end{array}$ & $\begin{array}{l}\text { Antes de salir de la casa general- } \\
\text { mente consumen líquidos (té, café } \\
\text { o mate) }\end{array}$ \\
Comen de pie o muy rápido & $\begin{array}{l}\text { No tienen horarios: se alimentan a } \\
\text { cualquier hora: } \\
\text { a media mañana comen snacks para } \\
\text { compensar la falta del desayuno }\end{array}$ \\
\end{tabular}

Almuerzo

Almuerzo casero (cazuela, legumbres)

Pocas ensaladas, por ejemplo un tipo de ensalada por día

Té u once/comida

Principalmente esta comida consta de té o leche, pan, y acompañamiento

Es la principal comida del día
Son selectivos para comer y prefieren alimentos secos, como tallarines, arroz y puré

Consumen pocas ensaladas y algunos días

Generalmente se adaptan a lo que comen sus hijos, que no comen saludable

Igual al Perfil 1

Cenan lo que quedó del almuerzo. Consumen un plato principal con ensaladas

Un subgrupo consume once/comida 
ejemplo, haciendo recetas. De todas formas, piensan que para aprender alimentación saludable, lo mejor es que los alumnos participen en talleres prácticos de cocina.

\section{DISCUSIÓN}

Existe consenso en Chile y en el mundo de la necesidad de educar en alimentación saludable a los niños y sus familias desde la escuela. La estrategia de "aprender la preferencia de alimentos saludables" ha sido una de las mejor evaluadas a nivel internacional, como lo demuestran publicaciones recientes (21). Para ello, es necesario tener un diagnóstico del consumo, hábitos alimentarios y habilidades culinarias que existen en la escuela, en este caso, en alumnos y padres de tercero a quinto año básico (8 a 11 años), semejante al que se realizó para niños de prekinder a segundo año básico (7). Al igual que en el diagnóstico anterior, en este trabajo se comprueba la mala alimentación de niños y padres, que no cumplen con las recomendaciones de las Guías Alimentarias chilenas (12), a pesar de que las conocen bien, con un bajo consumo de frutas, lácteos, pescado, agua y alto consumo de alimentos no saludables, especialmente en los padres (figura 1 ), lo cual preocupa porque son ellos los que modelan la alimentación de sus hijos.

En hábitos de consumo, $22 \%$ de los niños dice tomar doble desayuno y $33 \%$ doble almuerzo, lo cual preocupa en un país donde se está incrementando la obesidad infantil (22).
Esto debería ser considerado por la Junta Nacional de Auxilio Escolar y Becas (JUNAEB) que es la encargada del Programa de Alimentación Escolar (PAE) que entrega alimentos a los niños en las escuelas. Otro dato que debe ser tomado en consideración, es que $72,2 \%$ de los niños lleva dinero para comprar alimentos muy poco saludables en los kioskos, tal como se ha observado en estudios anteriores realizados por el INTA $(23,24)$.

En hábitos alimentarios, los padres dicen en su mayoría tener normas y comer juntos, pero $60 \%$ ve televisión cuando come, $95,6 \%$ toma once y $42 \%$ dice comer un plato de comida en la noche, con lo cual la once pasa a ser la principal comida del día, al igual que lo señalado en estudios anteriores (17-20).

En habilidades culinarias en los alumnos, un alto porcentaje dice hacer una ensalada, pero muy pocos preparan un plato de comida y si lo hacen cocinan arroz, fideos y huevos.

La mayoría de los padres dice hacer ensaladas pero un porcentaje muy bajo prepara platos de comida todos los días, lo cual coincide con el altísimo porcentaje que toma once en vez de comer en la noche. Los padres dicen preferir la comida casera y que cuando comen fuera del hogar la mayor parte lo hace en restaurantes y sólo $10 \%$ en locales de comida rápida, lo cual llama la atención, pero podría explicarse porque la mayoría son mujeres que viven en San Felipe.

Las encuestas muestran un alto acceso de padres e hijos a Ias TIC, en particular a redes sociales y a uso del computador, lo

TABLA 5.

Hábitos de alimentación los fines de semana de los apoderados según perfiles

\begin{tabular}{lll}
\hline \multicolumn{1}{c}{ Perfil 1 } & \multicolumn{1}{c}{ Perfil 2 } & \multicolumn{1}{c}{ Perfil 3} \\
\hline $\begin{array}{l}\text { M ejora sus hábitos alimenticios los fines } \\
\text { de semana porque: }\end{array}$ & $\begin{array}{l}\text { Empeora sus hábitos alimenticios el fin de } \\
\text { semana porque: }\end{array}$ & $\begin{array}{l}\text { La alimentación es la misma que los días de } \\
\text { semana. }\end{array}$ \\
$\begin{array}{l}\text { Aumentan el consumo de verduras y } \\
\text { frutas. }\end{array}$ & Baja la ingesta de frutas y verduras. & $\begin{array}{l}\text { Este grupo tiene buenos hábitos alimenticios } \\
\text { que mantiene durante la semana y fin de } \\
\text { semana. }\end{array}$ \\
$\begin{array}{l}\text { Tienen horarios definidos. } \\
\text { Se reúne toda la familia en la mesa a la } \\
\text { hora de cada comida. }\end{array}$ & $\begin{array}{l}\text { Visitan a familiares y comen alimentos altos } \\
\text { en calorías. }\end{array}$ & \\
Hay restricción de bebidas azucaradas. & $\begin{array}{l}\text { Aumenta el consumo de alimentos poco } \\
\text { saludables y de bebidas azucaradas. }\end{array}$ & \\
\hline
\end{tabular}

TABLA 6.

Almuerzo fuera del hogar de los apoderados según perfiles.

Perfil 1

Adultos llevan comida desde la casa, generalmente platos simples como arroz, fideos.

Hijos comen en el colegio y vuelven a almorzar nuevamente en la casa.

Consideran que la comida del casino es reducida y a veces no almuerzan porque no les gusta y compran en el kiosko.
Perfil 2

Los padres van a almorzar diariamente a sus casas, dado que trabajan cerca de sus hogares.

Hijos comen en el colegio y llegan a comer almuerzo $u$ once.
Perfil 3

Almuerzan en el trabajo, compran su comida, la que puede ser comida casera, sándwich, o chatarra. 
cual es muy importante de considerar para futuros programas de educación en nutrición.

Los grupos focales apoyan lo encontrado en las encuestas y son recomendados para conocer en profundidad hábitos y conductas frente a la alimentación propia y de sus hijos, por lo cual han sido utilizados para profundizar en temas nutricionales $(25,26)$.

Los padres reconocen sus malos hábitos alimentarios, poniéndose una nota baja (4,4 en escala de 1 a 7) señalando que sus principales barreras son la falta de tiempo para cocinar y el alto costo de la alimentación saludable. Al desagregarlos por grupos, llama la atención que los únicos que se alimentan en forma adecuada son aquellos que están enfermos, razón por la cual Ilevan una alimentación saludable por recomendación del médico o nutricionista. Es interesante el cambio de hábito los fines de semana, en donde aparece que aquellos que se alimentan mal durante la semana lo hacen mejor el fin de semana porque tienen más tiempo para cocinar.

En habilidades culinarias, los padres se califican con nota 6 , lo cual abre una buena perspectiva de trabajo educativo a través de talleres de cocina. Además, señalan que a los hijos también les gusta cocinar y que deberían existir talleres prácticos de cocina para ellos en las escuelas. Esto coincide con lo reportado por experiencias internacionales en otros países, donde para enseñar hábitos saludables, se han realizado intervenciones para enseñar a cocinar a los niños, como el "Cooking with kids program" $(25,27)$ y otras experiencias en América Latina (28). Estos programas reafirman una vez más que para poder cambiar hábitos desde la niñez, incorporando a las familias, es necesario realizar actividades prácticas de "aprender haciendo", que en el campo de la alimentación saludable se expresan en talleres de cocina y utilización de huertos escolares (29). Existen interesantes experiencias internacionales que apoyan los talleres prácticos de cocina en escuelas que deberían ser replicados en nuestro país, como la del chef Jamie Oliver, quien ha desarrollado programas en el Reino Unido y otros países (30), cuya efectividad está siendo evaluada actualmente.

En Chile, la existencia de una política de Estado en alimentación saludable para prevenir la obesidad infantil, es una necesidad imperiosa para enfrentar el tema desde diferentes ámbitos, dentro de los cuales la educación en alimentación saludable en escuelas normada desde el M inisterio de Educación, es uno de los más relevantes.

Agradecimientos: Este estudio fue financiado por el proyecto FONDECY T № 1140748 de Educación del Concurso Regular 2014: “Elaboración, aplicación y evaluación de un programa educativo en alimentación saludable, participativo y con uso de las Tecnologías de la Información y Comunicación (TIC) para profesores, alumnos de tercero, cuarto y quinto básico y sus familias". Investigador principal: Fernando Vio del Rio. Co-investigadora: Judith Salinas Cubillos. Duración: 3 años.

\section{RESUM EN}

El objetivo de este estudio fue describir el consumo, los hábitos alimentarios y las habilidades culinarias en alumnos de tercero a quinto año básico y de su familia por medio de encuestas y grupos focales para realizar intervenciones educativas en alimentación saludable en el ámbito escolar. Para ello se realizaron encuestas a alumnos y padres, que se complementaron con grupos focales. Según las encuestas, el consumo de alimentos saludables en alumnos y padres estuvo por debajo de lo recomendado en las Guías Alimentarias. El consumo de frutas, verduras y lácteos en los padres fue menor que en los hijos, pero consumieron menos alimentos no saludables. En hábitos, $22 \%$ de los niños tomaba doble desayuno y $33 \%$ doble almuerzo. Un $60 \%$ Ilevaba colación de la casa, pero $72 \%$ llevaba dinero destinado a compra de alimentos poco saludables. Los padres decían tener normas, pero un $60 \%$ veía televisión cuando comía, $96 \%$ tomaba once y $42,2 \%$ cenaba. En habilidades culinarias, los alumnos podían preparar alimentos simples, pero no un plato de comida. Las madres decían tener habilidades culinarias, pero cocinaban en forma ocasional y comida básica como papas, arroz y fideos. Los grupos focales confirmaron lo anterior, identificando tres perfiles de madres y diferenciando lo que comen en la semana con los fines de semana. Destaca el perfil de madres que se alimenta saludable, que eran las que han tenido un problema de salud. Las barreras para alimentarse bien son el alto costo y la falta de tiempo. Las encuestas y grupos focales mostraron un buen acceso de padres e hijos a las Tecnologías de la Información y Comunicación. Lo anterior demuestra el imperativo de contar con programas de educación nutricional prácticos para crear hábitos de alimentación saludable que consideren la situación actual de consumo, hábitos alimentarios y habilidades culinarias de los alumnos y sus padres.

Palabras clave: consumo alimentario, hábitos alimentarios, habilidades culinarias, educación nutricional, grupos focales, alumnos de tercero a quinto básico, padres.

\section{BIBLIOGRAFIA}

1. Escuelas mejores a través de la salud. Tercera Conferencia Europea sobre Escuelas Promotoras de la Salud. Vilna, Lituania, 15-17 de junio 2009.

2. Organización Panamericana de la Salud (OPS). Fortalecimiento de la Iniciativa Regional Escuelas Promotoras de la Salud: Estrategias y Líneas de Acción 2003-2012. Washington, D.C.: Serie Promoción de la Salud No 4 (74p) OPS, 2003.

3. Salinas J, Correa F, Vio F. M arco normativo para promover una alimentación saludable en escuelas básicas de Chile. Rev Chil Nutr. 2013; 40: 274-82.

4. Salinas J, Vio F. Programas de salud y nutrición sin política de estado: El caso de la promoción de salud escolar en Chile. Rev Chil Nutr. 2011; 38: 100-16.

5. Proyecto Fondecyt $\mathrm{N}$ ㅇ 1140748 “Elaboración, aplicación y evaluación de un programa educativo en alimentación saludable, participativo y con uso de Tecnologías de la Información y Comunicación (TIC) para profesores, alumnos de tercero, cuarto y quinto básico y sus familias". Concurso Fondecyt Regular 2014.

6. Kain J, Concha F, Salazar G, Leyton B, Rodríguez M P, Ceballos X, Vio F. Prevención de la obesidad en preescolares y escolares de escuelas municipales de una comuna de Santiago de Chile: Proyecto Piloto 2006. Arch Latinoam Nutr. 2009; 59:139-46.

7. Vio F, Salinas J, Lera L, González CG, Huenchupán C. Conocimiento y consumo alimentario en escolares, sus padres y profesores: Un análisis comparativo. Rev Chil Nutr. 2012; 39(3):34-9.

8. StataCorp. Stata Statistical Software: Release 12. College Station, TX: StataCorp. LP. 2011.

9. Patton, M. Q. Qualitative evaluation and research methods. Newbury Park, Inglaterra: Sage. 1991.

10. Aschidamini IM, SaupeR. Grupo focal: estratégia metodológica cualitativa: um ensaio teórico. Cogitate Enferm. 2004; 9:9-14. 
11. Lera L, Salinas J, Fretes F, Vio F. Elaboración de un instrumento para medir actitudes, conocimientos y prácticas alimentarias en familias chilenas de escolares de prebásica y básica. Nutr Hospitalaria 2013; 28:1957-66.

12. Olivares S, Zacarías I, González CG, Villalobos E. Proceso de formulación y validación de las guías alimentarias para la población chilena. Rev Chil Nutr. 2013; 40: 262-8.

13. Lera L, Fretes G, González CG, Salinas J, Vio F. Validación de un instrumento para evaluar consumo, hábitos y prácticas alimentarias en escolares de 8 a 11 años. Nutr Hosp. 2015; 31: 1980-91.

14. Olivares S, M orón C, Zacarías I, Andrade M, Vio F. Educación en nutrición en las escuelas básicas de Chile. Food Nutr Agricult (FAO) 2003; 33:64-9.

15. Krueger R.A. El grupo de discusión. Guía práctica para la investigación aplicada. M adrid. Pirámide. 1991.

16. Navarro P y Díaz C. "Análisis de Contenido", en "M étodos y Técnicas Cualitativas de Investigación en Ciencias Sociales. Delgado JM y Gutiérrez J. M adrid, España. Proyecto Editorial Síntesis Psicología. 1993.

17. Collect GfK. Encuesta de consumo de frutas y verduras en Chile. Abril 2008.

18. Adimark gFk. Obesidad. Creencias, actitudes y hábitos de los chilenos. Julio 2011.

19. M inisterio de Salud. Encuesta Nacional de Consumo Alimentario (ENCA). Chile 2010.

20. Salinas J, Lera L, González CG, Villalobos E, Vio F. Estilos de vida, alimentación y estado nutricional en trabajadores de la construcción de la Región M etropolitana de Chile. Rev M ed Chil. 2014; 142; 833-40.

21. Hawkes C, Smith TG, Jewell J, Wardle J, Hammond RA, Friel S, Thow AM, Kain J. Smart food policies for obesity prevention. www.thelancet.com. Published online February 18, 2015.
22. Junta Nacional de Auxilio Escolar y Becas (JUNAEB). M apa nutricional de Chile. Disponible en: http://bpt.junaeb. cl:8080/M apaNutricionalGx/ Accedido el 9 de julio de 2015.

23. Bustos N, Kain J, Leyton B, Vio F. Cambios en el patrón de consumo de alimentos en escolares chilenos con la implementación de un kiosko. Arch Latinoam N utr. 2011; 61:302-7

24. Bustos N, Kain J, Leyton B, Olivares S, Vio F. Colaciones habitualmente consumidas por niños de escuelas municipalizadas: M otivaciones para su elección. Rev Chil Nutr. 2010; 37: 178-83.

25. Lukas C, Cunningham-Sabo I. Qualitative investigation of the Cooking with Kids Program: Focus group interviews with fourth-grade students, teachers and food educators. J Educ Nutr Behav. 2011; 43: 517-24.

26. Cervato-M ancuso AM, M oraes da Silva G, Pava Cárdenas A. Grupos focal con nutricionistas que actúan en la alimentación escolar: el menú como elemento pedagógico. Rev Chil Nutr. 2013; 40: 250-5.

27. Walters LM, Stacey JE. Focus on food: development of the Cooking with Kids experimental nutrition education curriculum. J Educ Nutr Behav. 2009; 41: 371-3.

28. De Campo M L, Vara M essler M, Navarro A. Educación Alimentaria Nutricional (EAN) de la enseñanza primaria municipal de Córdoba. Una experiencia en investigaciónacción participativa (IAP). Año 2008 Diaeta (B.Aires) 2010; 28:15-22.

29. Davis JN, Spaniol M R, Somerset S. Sustenance and sustainability: maximizing the impact of school gardens on health outcomes. Public Health Nutr. 2015;23:1-10.

30. Flegs A, Herbert J, Gibbs L, Swinburn B, Keating C, Waters E, M oodie M. M ethods for the evaluation of Jamie O liver M inistry of Food program, Australia. BM C Public Health 2013; 13:411. 